\title{
Fatores associados à insatisfação com a Imagem Corporal em adultos: análise seccional do ELSA-Brasil
}

\author{
Factors associated with body image dissatisfaction in adults: \\ a cross-sectional analysis of the ELSA-Brasil Study
}

Liliane da Silva Albuquerque (https://orcid.org/0000-0001-8182-4906) ${ }^{1}$

Rosane Harter Griep (https://orcid.org/0000-0002-6250-2036) ${ }^{2}$

Estela M. L. Aquino (https://orcid.org/0000-0002-8204-1249) ${ }^{3}$

Letícia de Oliveira Cardoso (https://orcid.org/0000-0003-1312-1808) ${ }^{4}$

Dóra Chor (https://orcid.org/0000-0001-9981-6402) ${ }^{4}$

Maria de Jesus Mendes da Fonseca (https://orcid.org/0000-0002-5319-5513) ${ }^{4}$
${ }^{1}$ Secretaria Municipal de Educação da cidade do Rio de Janeiro. Escola Municipal Lino Martins da Silva. R. Teixeira Ribeiro 1000 bloco 1, Campus Maré Bonsucesso. 21044-251 Rio de Janeiro RJ Brasil. lilianesalbuquerque@ gmail.com

${ }^{2}$ Laboratório de Educação em Ambiente e Saúde, Instituto Oswaldo cruz, Fundação Oswaldo Cruz. Rio de Janeiro RJ Brasil. ${ }^{3}$ Instituto de Saúde Coletiva, Universidade Federal da Bahia. Salvador BA Brasil. ${ }^{4}$ Departamento de Epidemiologia e Métodos Quantitativos em Saúde, Escola Nacional de Saúde Pública Sergio Arouca, Fundação Oswaldo Cruz Rio de Janeiro RJ Brasil.

\begin{abstract}
The aim of this article is to assess the association between body image dissatisfaction and sociodemographic and health behaviors, according to sex. Data were analyzed for 6,289 women and 5,188 men (35-59 years), participants in the baseline of the Longitudinal Study of Adult Health (ELSA-Brasil), using multinomial regression. The odds of dissatisfaction due to feeling underweight were higher among in women with low schooling and those who only consumed fruit weekly. Moderate physical activity reduced this type of dissatisfaction by $50 \%$. Higher odds of dissatisfaction due to overweight were seen in married women, those who practiced light physical activity, and former smokers. Men with secondary schooling and excessive alcohol consumption showed 50\% higher odds of dissatisfaction due to underweight, while light or moderate physical activity increased the odds by 75\% and 94\%, respectively. Among men, light and moderate physical activity were also associated with increased odds of dissatisfaction due to overweight. These findings corroborate that unhealthy habits and behaviors can influence body image dissatisfaction with different patterns between women and men.
\end{abstract}

Keywords Body image, Risk factors, Distributions by sex
Resumo O objetivo deste artigo é avaliar a associação entre imagem corporal e variáveis sociodemográficas e comportamentos de saúde, por sexo masculino e feminino. Analisou-se dados de 6.289 mulheres e 5.188 homens, entre 35 e 59 anos, participantes da linha de base do Estudo Longitudinal de Saúde do Adulto (ELSA-Brasil) com a utilização da regressão multinomial. Entre as mulheres, a chance de insatisfação por se sentirem magras foi mais alta entre as com menor escolaridade $e$ as que consumiam frutas semanalmente. A prática de atividade fisica moderada reduziu em $50 \%$ a chance deste tipo de insatisfação. Graus mais elevados de insatisfação por estar acima do peso foram observados entre as casadas, as que praticam atividade fisica leve e as ex-fumantes. Homens com nível médio de escolaridade e consumo excessivo de álcool tiveram em torno de 50\% de chances mais elevadas de insatisfação por baixo peso, posto que a prática de atividade física leve ou moderada elevou essa chance em $75 \%$ e $94 \%$, respectivamente. Entre os homens, a prática de atividade física leve e moderada também aumentam a chance de insatisfação por estarem acima do peso. Esses achados contribuem para reforçar que hábitos e comportamentos não saudáveis podem influenciar a insatisfação da imagem corporal com padrões diferenciados, segundo sexo.

Palavras-chave Imagem corporal, Fatores de risco, Distribuições por sexo 


\section{Introdução}

Imagem Corporal (IC) refere-se às percepções, pensamentos e sentimentos do indivíduo sobre a aparência do seu próprio corpo ${ }^{1,2}$. É influenciada por muitos fatores tais como a mídia, o humor, as emoções, a autoestima e as influências do meio sociocultural de vida das pessoas ${ }^{3,4}$. Além disso, é imbuída de concepções históricas acerca do padrão de beleza, experiências adquiridas, características físicas ao longo da vida e influências da própria personalidade ${ }^{4,5}$. A imagem corporal pode variar ao longo da vida, moldando e influenciando as ações dos indivíduos em função daquilo que se considera "normal" e aceitável de acordo com o ambiente social em que estão inseridos' ${ }^{1}$.

A vida adulta proporciona mudanças, fisiológicas e sociais, que afetam a relação do indivíduo com o seu corpo. As alterações fisiológicas estão atreladas ao processo de amadurecimento natural. No que se refere aos aspectos sociais, ocorrem mudanças no padrão de vida, no mundo do trabalho, entre outras questões ${ }^{6,7}$.

A percepção da imagem corporal pode ser avaliada por um gradiente amplo desde percepções positivas, satisfação e aceitação do formato do corpo até sentimentos negativos que geram sofrimento com a aparência física ${ }^{8}$. No entanto, os estudos têm focado nos efeitos relacionados à insatisfação e experiências negativas com a imagem corporal $^{3-5,8}$. A insatisfação com a imagem corporal (body dissatisfaction) ocorre quando a imagem percebida do corpo não é compatível com aquela idealizada, e pode gerar estresse e sofrimento, influenciar o humor, os comportamentos em saúde (especialmente na dieta e na atividade física) e a saúde mental, o que afeta negativamente a qualidade de vida ${ }^{4,8}$.

A insatisfação com a imagem corporal difere entre homens e mulheres e apresenta diferenças biológicas marcantes relacionadas à aparência $\mathrm{e}$ às funções corporais. Entretanto, o ambiente social exerce forte impacto na avaliação do próprio corpo, por exemplo, no ideal midiático de corpo feminino associado à magreza e de corpo com musculatura desenvolvida para os homens ${ }^{4}$. De fato, os estudos têm mostrado que a insatisfação por desejar ser mais magra do que realmente se percebem é mais prevalente entre as mulheres do que em homens, com ocorrência em mulheres de todas as idades ${ }^{4,9}$. Entre os homens, o estudo dos efeitos da insatisfação é mais recente e tem apontado, mais frequentemente, para a insatisfação de não possuirem um corpo mais musculoso do que o real ${ }^{10,11}$.
O interesse no estudo da imagem corporal, tanto no que se refere aos seus fatores determinantes quanto a sua consequência sobre a saúde tem sido crescente ${ }^{4}$. Alguns artigos têm abordado aspectos como transtornos alimentares, depressão, atividade física e outras questões ligadas ao constructo multidimensional e complexo que é a insatisfação com a imagem corporal $^{8,12,13}$. No Brasil, embora ainda predominem estudos com adolescentes e adultos jovens universitários ${ }^{9,14}$, publicações mais recentes abordam a associação da insatisfação com a imagem corporal e o estado nutricional, além de comportamentos de saúde, especialmente atividade física em adultos ${ }^{15-19}$. No que foi possível avaliar, não se identificou estudo nacional que tenha avaliado a prevalência do tipo de insatisfação (por peso abaixo ou mais alto do que o desejado) em homens em mulheres e sua associação com características sociodemográficas e comportamentos de saúde em amostra abrangente e isenta de transtornos específicos de alimentação.

Este artigo tem o objetivo de avaliar a prevalência do tipo de insatisfação com a imagem corporal, por baixo peso ou por excesso de peso, e a sua associação com fatores socioeconômicos e comportamentais entre homens e mulheres, na faixa etária entre 35 a 59 anos, participantes do Estudo Longitudinal de Saúde do Adulto (ELSA -Brasil).

\section{Métodos}

Analisou-se os dados da linha de base (20082010) do Estudo Longitudinal de Saúde do Adulto (ELSA-Brasil), uma coorte de trabalhadores composta de 15.105 servidores públicos ativos ou aposentados, com idade entre 35 e 74 anos, de instituições localizadas em seis diferentes estados do Brasil (Bahia, Espírito Santo, Minas Gerais, Rio de Janeiro, São Pauloe Rio Grande do Sul). Maiores detalhes sobre o estudo podem ser obtidos em outra publicação ${ }^{20}$. No presente estudo, foram incluídos os dados dos participantes com idade entre 35 e 59 anos $(\mathrm{N}=11.842)$, sendo que cerca de $97 \%$ (11.477) apresentaram todos os dados de interesse (6.289 mulheres e 5.188 homens).

Obteve-se a variável dependente Imagem Corporal por meio da escala de figuras de silhuetas, desenvolvida e validada por Kakeshita et al. ${ }^{21}$, composta por 15 figuras de silhuetas para cada sexo. As figuras são apresentadas da mais magra para a mais obesa e, após a exposição de todas, 
ao sujeito é solicitado escolher duas figuras: uma que melhor represente o seu corpo atual e outra que melhor represente o corpo que ele gostaria de ter. A avaliação da confiabilidade da escala foi elevada. Os valores do coeficiente de correlação intraclasse foram superiores a 0,90 , em sua maioria $^{22}$.

Realizou-se a classificação da insatisfação com a Imagem Corporal através de um escore, calculado pela subtração entre o algarismo indicado na figura que melhor representa o "corpo atual" selecionada pelo participante e o algarismo presente na figura que representa o corpo "que gostaria de ter" podendo variar de menos 15 até 15. Classificou-se o desfecho, adaptando para escala da Kakeshita et al. ${ }^{21}$ a categorização utilizada na literatura com a escala de Stunkard ${ }^{15,23,24}$ em 'satisfeito' (escore igual a zero, ou seja, o participante escolheu a mesma figura para o corpo percebido e para o corpo ideal); 'insatisfeito com a imagem corporal por baixo peso (BP)' (escore negativo, escolha de uma silhueta ideal maior do que a atual), e insatisfeito com a imagem corporal por excesso de peso (EP), escore positivo por escolher uma silhueta ideal menor do que a atual.

As covariáveis foram: idade (contínua), nível de escolaridade (ensino fundamental, médio e superior), renda per capita (em tercis), situação conjugal (solteiro, separado/viúvo, e casado ou em união), prática de atividade física, avaliada por meio dos blocos de deslocamento e de atividade física de lazer do Questionário Internacional de Atividade Física (IPAQ) versão longa ${ }^{25}$, considerando as categorias atividade física leve, moderada e forte ${ }^{26}$. Mensurou-se o consumo de frutas e hortaliças por meio de duas perguntas: Com que frequência o(a) Sr. (a) costuma comer frutas? e Com que frequência o(a) Sr.(a) costuma comer verduras ou legumes crus, cozidos ou refogados, sem incluir batatas, mandioca/aipim, inhame e cará? Apresentado em oito opções de resposta que foram agrupadas em: alto consumo (duas ou mais vezes por dia), consumo diário (uma vez por dia ou cinco a seis vezes por semana), consumo semanal (duas a quatro vezes por semana) e consumo raro (uma vez por semana ou menos ${ }^{27}$. Categorizou-se o hábito de fumar em não fumante, ex-fumante e fumante atual e o consumo de álcool em não consome bebidas alcoólicas atualmente, consumo moderado ( $<140$ gramas de álcool por semana para as mulheres e $<210$ gramas para os homens) e consumo excessivo ( $\geq$ 140 gramas mulheres e $\geq 210$ gramas homens) ${ }^{28}$.

$\mathrm{Na}$ análise estatística, calculou-se as frequências absolutas e relativas para a análise des- critiva. Na modelagem empregou-se o modelo multinomial que foi estimado nas análises simples e múltiplas, ajustada para estimação da razão de chance (OR), com intervalos de $95 \%$ de confiança. Realizaram-se análises separadas por sexo, com base nos resultados da literatura que apresentam direções diferentes entre homens e mulheres. Construiu-se modelos pela introdução das variáveis que apresentaram na análise bruta do valor de $\mathrm{p}$ associado ao parâmetro $<0,10$. No primeiro bloco, considerou-se as variáveis sociodemográficas e, no segundo bloco, as variáveis comportamentais, em que aquelas que permaneceram significativas no primeiro e segundo blocos foram agrupadas no modelo final. Utilizou-se o Índice de Massa Corporal (IMC) apenas para descrever a população do estudo, considerando as categorias de classificação do estado nutricional da Organização Mundial de Saúde $(\mathrm{OMS})^{29}$. Optou-se por não ajustar os modelos pelo IMC, tendo em vista que a escala de silhuetas utilizada foi construída com base nesta medi$\mathrm{da}^{21}$, o que poderia resultar em um super-ajuste.

\section{Considerações éticas}

A Comissão Nacional de Ética em Pesquisa (CONEP) e os Comitês de Ética e Pesquisa (CEPS) de todas as instituições envolvidas aprovaram o estudo: Universidade de São Paulo, Universidade Federal de Minas Gerais, Fundação Oswaldo Cruz, Universidade Federal do Espírito Santo, Universidade Federal da Bahia e Universidade Federal do Rio Grande do Sul.

\section{Resultados}

As médias de idade foram semelhantes entre homens e mulheres. Comparadas aos homens, as mulheres referiram mais frequentemente insatisfação por excesso de peso (EP) e menos por baixo peso (BP), embora o sobrepeso tenha sido mais frequente entre os homens. As mulheres apresentaram escolaridade e renda mais alta, frequência mais alta de solteiras ou separadas/viúvas, e um pouco mais baixa para atividade física forte e moderada, além de apresentarem menor consumo de bebidas alcoólicas e história de tabagismo atual ou no passado menores em relação aos homens. Elas ainda relataram consumir frutas e hortaliças mais frequentemente do que os homens (Tabela 1).

Ao considerar as análises brutas, observou-se que, entre as mulheres, ter ensino fundamental, 
Tabela 1. Distribuição proporcional das variáveis sociodemográficas, hábitos e comportamentos segundo sexo. Estudo ELSA-Brasil, 2008-2010.

\begin{tabular}{|c|c|c|c|c|}
\hline \multirow{2}{*}{ Variáveis } & \multicolumn{2}{|c|}{ Mulheres } & \multicolumn{2}{|c|}{ Homens } \\
\hline & $\mathbf{n}$ & $\%$ & $\mathbf{n}$ & $\%$ \\
\hline População & 6.289 & 54,8 & 5.188 & 45,2 \\
\hline \multicolumn{5}{|l|}{ Imagem corporal $^{\star}$} \\
\hline Satisfeito & 656 & 10,4 & 977 & 18,8 \\
\hline Insatisfeito BP & 275 & 4,4 & 589 & 11,4 \\
\hline Insatisfeito EP & 5.358 & 85,2 & 3.622 & 69,8 \\
\hline \multicolumn{5}{|l|}{$\mathrm{IMC}^{*}$} \\
\hline Eutrófico & 2.496 & 39,7 & 1.715 & 33,1 \\
\hline Baixo peso & 61 & 1,0 & 55 & 1,1 \\
\hline Sobrepeso & 2.217 & 35,3 & 2.321 & 44,7 \\
\hline Obesidade & 1.515 & 24,1 & 1.097 & 21,1 \\
\hline \multicolumn{5}{|l|}{ Escolaridade $^{\star}$} \\
\hline Ensino superior & 3.485 & 55,4 & 2.500 & 48,2 \\
\hline Ensino médio & 2.381 & 37,9 & 1.918 & 37,0 \\
\hline Ensino fundamental & 423 & 6,7 & 770 & 14,8 \\
\hline \multicolumn{5}{|l|}{ Renda per capita* } \\
\hline Alta & 2.027 & 32,2 & 1.556 & 30,0 \\
\hline Média & 2.226 & 35,4 & 1.623 & 31,3 \\
\hline Baixa & 2.036 & 32,4 & 2.009 & 38,7 \\
\hline \multicolumn{5}{|l|}{ Situação Conjugal ${ }^{*}$} \\
\hline Solteiro & 890 & 14,2 & 284 & 5,5 \\
\hline Separado/Viúvo & 1.887 & 30,0 & 692 & 13,3 \\
\hline Casado & 3.512 & 55,8 & 4.212 & 81,2 \\
\hline \multicolumn{5}{|l|}{ Atividade Física ${ }^{*}$} \\
\hline Forte & 472 & 7,5 & 598 & 11,5 \\
\hline Moderada & 732 & 11,6 & 709 & 13,7 \\
\hline Fraca & 5.085 & 80,9 & 3.881 & 74,8 \\
\hline \multicolumn{5}{|l|}{ Consumo de Frutas ${ }^{\star}$} \\
\hline Alto & 1.589 & 25,3 & 731 & 14,1 \\
\hline Diário & 2.841 & 45,2 & 1.998 & 38,5 \\
\hline Semanal & 1.222 & 19,4 & 1.447 & 27,9 \\
\hline Raro & 637 & 10,1 & 1.012 & 19,5 \\
\hline \multicolumn{5}{|l|}{ Consumo de Hortaliças ${ }^{\star}$} \\
\hline Alto & 1.038 & 16,5 & 628 & 12,1 \\
\hline Diário & 3.183 & 50,6 & 2.208 & 42,6 \\
\hline Semanal & 1.344 & 21,4 & 1.392 & 26,8 \\
\hline Raro & 724 & 11,5 & 960 & 18,5 \\
\hline \multicolumn{5}{|l|}{ Consumo de Álcool ${ }^{*}$} \\
\hline Não consome & 3.722 & 59,2 & 1.853 & 35,7 \\
\hline Consumo moderado & 2.340 & 37,2 & 2.699 & 52,0 \\
\hline Consumo excessivo & 227 & 3,6 & 636 & 12,3 \\
\hline \multicolumn{5}{|l|}{ Hábito de Fumar* } \\
\hline Nunca fumou & 3.893 & 61,9 & 2.769 & 53,4 \\
\hline Ex-fumante & 1.567 & 24,9 & 1.638 & 31,6 \\
\hline Fumante & 829 & 13,2 & 781 & 15,1 \\
\hline
\end{tabular}

consumir frutas semanalmente, hortaliças raramente e ser fumante, representou uma chance cerca de duas vezes mais elevada de desejar uma silhueta corporal mais volumosa. Com relação à insatisfação com a imagem corporal por se sentirem com excesso de peso, as separadas/viúvas ou casadas, com prática de atividade física leve e as ex-fumantes apresentaram chance aproximadamente 1,5 vezes mais elevada dessa insatisfação (Tabela 2).

Entre os homens, a chance de estarem insatisfeitos com a imagem corporal por se sentirem magros foi cerca de 1,5 vezes mais para aqueles que tinham ensino médio, renda per capita baixa e consumiam álcool excessivamente e de 1,8 vezes para a prática de atividade física leve/moderada. Os fatores que aumentaram a chance de insatisfação por excesso de peso foram apresentar atividade física leve/moderada, consumo de frutas e hortaliças semanal, consumo excessivo de álcool e ser ex-fumante. Em contrapartida, escolaridade e renda mais baixas e hábito de fumar atual foram fatores que diminuíram a chance de se sentir acima do peso entre os homens (Tabela 3).

No modelo final (Tabela 4), entre as mulheres, observou-se uma chance aproximadamente duas vezes mais elevada de insatisfação por se sentirem magras dentre aquelas com menor escolaridade e consumo de fruta apenas semanal. Enquanto que a prática de atividade física moderada reduziu em torno de $50 \%$ a chance deste tipo de insatisfação. Já entre os homens, os fatores que aumentaram em torno de $50 \%$ a chance de insatisfação por se sentirem magros, foram escolaridade de nível médio e consumo de álcool excessivo, já a prática atividade física leve ou moderada elevou essa chance em $75 \%$ e 94\%, respectivamente.

Com relação à insatisfação com a imagem corporal por se sentir acima do peso (Tabela 5), as mulheres casadas, com prática de atividade física leve ou ex-fumantes apresentaram chance em torno de 50\% mais elevada de insatisfação. Entre os homens, não apenas a prática de atividade física leve, mas também a prática moderada aumentaram a chance de insatisfação. O hábito de fumar também foi importante no contexto masculino, apresentando chance mais elevada de insatisfação entre os ex-fumantes e mais baixa entre os fumantes atuais. Além destes fatores, também se observou uma chance aproximadamente de $90 \%$ mais elevada de insatisfação relacionada ao consumo excessivo de álcool. 
Tabela 2. Associação bruta entre as variáveis sociodemográficas e de comportamento e Imagem Corporal para as mulheres. Estudo ELSA-Brasil, 2008-2010.

\begin{tabular}{|c|c|c|c|c|c|c|c|c|}
\hline \multirow{2}{*}{$\begin{array}{l}\text { Mulheres } \\
\text { Variáveis }\end{array}$} & \multicolumn{2}{|c|}{ Satisfeitas } & \multicolumn{3}{|c|}{ Insatisfeitas com a IC BP } & \multicolumn{3}{|c|}{ Insatisfeitas com a IC EP } \\
\hline & $\mathbf{n}$ & $\%$ & $\mathbf{n}$ & $\%$ & $\begin{array}{l}\text { OR bruta } \\
\text { (IC 95\%) }\end{array}$ & $\mathbf{n}$ & $\%$ & $\begin{array}{l}\text { OR bruta } \\
\text { (IC 95\%) }\end{array}$ \\
\hline \multicolumn{9}{|l|}{ Escolaridade } \\
\hline Ensino superior & 386 & 11,1 & 136 & 3,9 & 1 & 2.963 & 85,0 & 1 \\
\hline Ensino médio & 230 & 9,7 & 108 & 4,5 & $1,33(0,99-1,8)$ & 2.043 & 85,8 & $1,16(0,97-1,38)$ \\
\hline Ensino fundamental & 40 & 9,5 & 31 & 7,3 & $2,2(1,32-3,66)^{* *}$ & 352 & 83,2 & $1,15(0,81-1,62)$ \\
\hline \multicolumn{9}{|l|}{ Renda per capita } \\
\hline Alta & 225 & 11,1 & 84 & 4,1 & 1 & 1.718 & 84,8 & 1 \\
\hline Média & 241 & 10,8 & 90 & 4,0 & $1,0(0,71-1,42)$ & 1.895 & 85,1 & $1,03(0,85-1,25)$ \\
\hline Baixa & 190 & 9,3 & 101 & 5,0 & $1,42(1,0-2,02)$ & 1.745 & 85,7 & $1,2(0,98-1,48)$ \\
\hline \multicolumn{9}{|l|}{ Situação Conjugal } \\
\hline Solteiro & 121 & 13,6 & 49 & 5,5 & 1 & 720 & 80,9 & 1 \\
\hline Separado/Viúvo & 199 & 10,5 & 90 & 4,8 & $1,12(0,74-1,69)$ & 1.598 & 84,7 & $1,35(1,06-1,72)^{\star}$ \\
\hline Casado & 336 & 9,6 & 136 & 3,9 & $1,0(0,68-1,47)$ & 3.040 & 86,6 & $1,52(1,22-1,9)^{\star * *}$ \\
\hline \multicolumn{9}{|l|}{ Atividade Física } \\
\hline Forte & 64 & 13,6 & 22 & 4,7 & 1 & 386 & 81,8 & 1 \\
\hline Moderada & 110 & 15,0 & 19 & 2,6 & $0,5(0,25-1,0)$ & 603 & 82,4 & $0,91(0,65-1,27)$ \\
\hline Fraca & 482 & 9,5 & 234 & 4,6 & $1,41(0,85-2,35)$ & 4.369 & 85,9 & $1,5(1,14-1,99)^{\star *}$ \\
\hline \multicolumn{9}{|l|}{ Consumo de Frutas } \\
\hline Alto & 156 & 9,8 & 47 & 3,0 & 1 & 1.386 & 87,2 & 1 \\
\hline Diário & 311 & 10,9 & 120 & 4,2 & $1,28(0,87-1,89)$ & 2.410 & 84,8 & $0,87(0,71-1,07)$ \\
\hline Semanal & 112 & 9,2 & 73 & 6,0 & $2,16(1,39-3,36)^{* * *}$ & 1.037 & 84,9 & $1,04(0,81-1,35)$ \\
\hline Raro & 77 & 12,1 & 35 & 5,5 & $1,51(0,9-2,53)$ & 525 & 82,4 & $0,77(0,57-1,03)$ \\
\hline \multicolumn{9}{|l|}{ Consumo de Hortaliças } \\
\hline Alto & 106 & 10,2 & 37 & 3,6 & 1 & 895 & 86,2 & 1 \\
\hline Diário & 348 & 10,9 & 121 & 3,8 & $1,0(0,65-1,53)$ & 2.714 & 85,3 & $0,92(0,73-1,16)$ \\
\hline Semanal & 131 & 9,7 & 72 & 5,4 & $1,57(0,98-2,52)$ & 1.141 & 84,9 & $1,03(0,79-1,35)$ \\
\hline Raro & 71 & 9,8 & 45 & 6,2 & $1,82(1,07-3,08)^{\star}$ & 608 & 84,0 & $1,01(0,74-1,39)$ \\
\hline \multicolumn{9}{|l|}{ Consumo de Álcool } \\
\hline Não consome & 385 & 10,3 & 171 & 4,6 & 1 & 3.166 & 85,1 & 1 \\
\hline Consumo moderado & 251 & 10,7 & 98 & 4,2 & $0,88(0,65-1,18)$ & 1.991 & 85,1 & $0,96(0,81-1,14)$ \\
\hline Consumo excessivo & 20 & 8,8 & 6 & 2,6 & $0,68(0,27-1,71)$ & 201 & 88,5 & $1,22(0,76-1,96)$ \\
\hline \multicolumn{9}{|l|}{ Hábito de Fumar } \\
\hline Nunca fumou & 437 & 11,2 & 169 & 4,3 & 1 & 3.287 & 84,4 & 1 \\
\hline Ex-fumante & 120 & 7,7 & 44 & 2,8 & $0,95(0,64-1,4)$ & 1.403 & 89,5 & $1,55(1,26-1,92)^{\star * *}$ \\
\hline Fumante & 99 & 11,9 & 62 & 7,5 & $1,62(1,13-2,33)^{\star *}$ & 668 & 80,6 & $0,9(0,71-1,13)$ \\
\hline
\end{tabular}

\section{Discussão}

A prevalência de insatisfação foi elevada e diferiu de acordo com o sexo e o tipo de insatisfação. A por excesso de peso foi mais elevada entre as mulheres e a insatisfação por baixo peso foi mais frequente entre os homens. Em três estudos re- alizados no Brasil ${ }^{15,24,30}$, as prevalências de insatisfação por se sentir acima do peso, entre mulheres, foram menores, em torno de $65 \%$. Entre os homens os achados são mais divergentes com as prevalências, com variação de $26 \%$ a $46 \%$. A diferença entre as prevalências encontradas pode estar relacionada à utilização de diferentes ins- 
Tabela 3. Associação bruta entre as variáveis sociodemográficas e de comportamento e Imagem Corporal para os homens. Estudo ELSA-Brasil, 2008-2010.

\begin{tabular}{|c|c|c|c|c|c|c|c|c|}
\hline \multirow{2}{*}{$\begin{array}{l}\text { Homens } \\
\text { Variáveis }\end{array}$} & \multicolumn{2}{|c|}{ Satisfeitos } & \multicolumn{3}{|c|}{ Insatisfeitos com a IC BP } & \multicolumn{3}{|c|}{ Insatisfeitos com a IC EP } \\
\hline & $\mathbf{n}$ & $\%$ & $\mathbf{n}$ & $\%$ & $\begin{array}{c}\text { OR bruta } \\
\text { (Intervalo de } \\
\text { Confiança 95\%) }\end{array}$ & $\mathbf{n}$ & $\%$ & $\begin{array}{c}\text { OR bruta } \\
\text { (Intervalo de } \\
\text { Confiança 95\%) }\end{array}$ \\
\hline \multicolumn{9}{|l|}{ Escolaridade } \\
\hline Ensino superior & 439 & 17,6 & 222 & 8,9 & 1 & 1.839 & 73,6 & 1 \\
\hline Ensino médio & 342 & 17,8 & 263 & 13,7 & $1,52(1,21-1,91)^{* * *}$ & 1.313 & 68,5 & $0,92(0,78-1,07)$ \\
\hline Ensino fundamental & 196 & 25,5 & 104 & 13,5 & $1,05(0,79-1,4)$ & 470 & 61,0 & $0,57(0,47-0,7)^{\star * *}$ \\
\hline \multicolumn{9}{|l|}{ Renda per capita } \\
\hline Alta & 280 & 18,0 & 137 & 8,8 & 1 & 1.139 & 73,2 & 1 \\
\hline Média & 291 & 17,9 & 170 & 10,5 & $1,19(0,9-1,58)$ & 1.162 & 71,6 & $0,98(0,82-1,18)$ \\
\hline Baixa & 406 & 20,2 & 282 & 14,0 & $1,42(1,1-1,83)^{\star \star}$ & 1.321 & 65,8 & $0,8(0,67-0,95)^{*}$ \\
\hline \multicolumn{9}{|l|}{ Situação Conjugal } \\
\hline Solteiro & 52 & 18,3 & 43 & 15,1 & 1 & 189 & 66,5 & 1 \\
\hline Separado/Viúvo & 134 & 19,4 & 98 & 14,2 & $0,88(0,55-1,43)$ & 460 & 66,5 & $0,94(0,66-1,36)$ \\
\hline Casado & 791 & 18,8 & 448 & 10,6 & $0,68(0,45-1,04)$ & 2.973 & 70,6 & $1,03(0,75-1,42)$ \\
\hline \multicolumn{9}{|l|}{ Atividade Física } \\
\hline Forte & 164 & 27,4 & 60 & 10,0 & 1 & 374 & 62,5 & 1 \\
\hline Moderada & 142 & 20,0 & 95 & 13,4 & $1,83(1,23-2,71)^{\star *}$ & 472 & 66,6 & $1,46(1,12-1,89)^{\star *}$ \\
\hline Fraca & 671 & 17,3 & 434 & 11,2 & $1,77(1,28-2,43)^{* * *}$ & 2.776 & 71,5 & $1,81(1,48-2,22)^{\star \star *}$ \\
\hline \multicolumn{9}{|l|}{ Consumo de Frutas } \\
\hline Alto & 159 & 21,8 & 75 & 10,3 & 1 & 497 & 68,0 & 1 \\
\hline Diário & 370 & 18,5 & 220 & 11,0 & $1,26(0,91-1,74)$ & 1.408 & 70,5 & $1,22(0,98-1,51)$ \\
\hline Semanal & 251 & 17,3 & 165 & 11,4 & $1,39(0,99-1,95)$ & 1.031 & 71,3 & $1,31(1,05-1,65)^{*}$ \\
\hline Raro & 197 & 19,5 & 129 & 12,7 & $1,39(0,98-1,98)$ & 686 & 67,8 & $1,11(0,88-1,41)$ \\
\hline \multicolumn{9}{|l|}{ Consumo de Hortaliças } \\
\hline Alto & 136 & 21,7 & 71 & 11,3 & 1 & 421 & 67,0 & 1 \\
\hline Diário & 415 & 18,8 & 221 & 10,0 & $1,02(0,73-1,42)$ & 1.572 & 71,2 & $1,22(0,98-1,53)$ \\
\hline Semanal & 242 & 17,4 & 169 & 12,1 & $1,34(0,94-1,89)$ & 981 & 70,5 & $1,31(1,03-1,66)^{*}$ \\
\hline Raro & 184 & 19,2 & 128 & 13,3 & $1,33(0,92-1,92)$ & 648 & 67,5 & $1,14(0,88-1,47)$ \\
\hline \multicolumn{9}{|l|}{ Consumo de Álcool } \\
\hline Não consome & 382 & 20,6 & 227 & 12,3 & 1 & 1.244 & 67,1 & 1 \\
\hline Consumo moderado & 511 & 18,9 & 283 & 10,5 & $0,93(0,75-1,16)$ & 1.905 & 70,6 & $1,14(0,98-1,33)$ \\
\hline Consumo excessivo & 84 & 13,2 & 79 & 12,4 & $1,58(1,12-2,24)^{\star \star}$ & 473 & 74,4 & $1,73(1,33-2,24)^{\star * *}$ \\
\hline \multicolumn{9}{|l|}{ Hábito de Fumar } \\
\hline Nunca fumou & 527 & 19,0 & 322 & 11,6 & 1 & 1.920 & 69,3 & 1 \\
\hline Ex-fumante & 268 & 16,4 & 128 & 7,8 & $0,78(0,61-1,01)$ & 1.242 & 75,8 & $1,27(1,08-1,5)^{\star *}$ \\
\hline Fumante & 182 & 23,3 & 139 & 17,8 & $1,25(0,96-1,62)$ & 460 & 58,9 & $0,69(0,57-0,84)^{\star * *}$ \\
\hline
\end{tabular}

trumentos para avaliação da imagem corporal e também à diferença de idade, pois a faixa etária estudada era maior (35 e 59 anos), e a média de idade 48 anos. Sabe-se que com o aumento da idade ocorre uma tendência de elevação do IMC $^{31}$, o que pode estar relacionado a prevalências mais altas. Por outro lado, alguns estudos apontam ${ }^{32}$, ${ }^{33}$ que com o passar da idade, o modelo de beleza do corpo jovem vai diminuindo e existe uma adaptação das expectativas para o alcance deste modelo. Entretanto, por nossa amostra constituir de indivíduos com alto nível de escolaridade e inseridos no mercado de trabalho, esse desejo de alcançar corpos menos volumosos pode ainda estar presente.

Observou-se associação com o nível de escolaridade foi observada para os dois tipos de insatisfação com a imagem corporal, entretanto, 


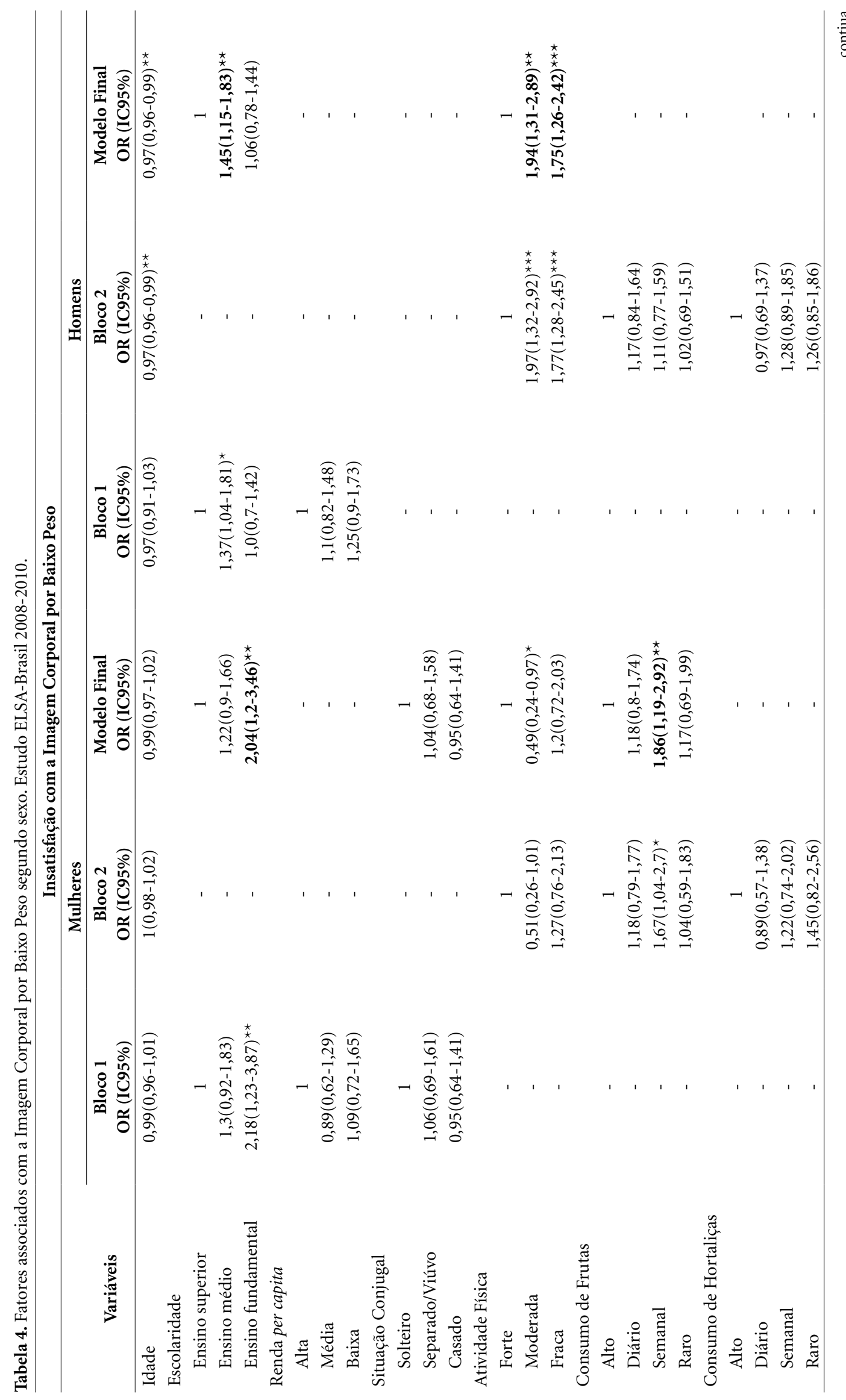


se comportando de forma inversa. Observou-se também chances mais elevadas de insatisfação com a imagem corporal por baixo peso foram observadas para a escolaridade mais baixa, e para a insatisfação com a imagem corporal por excesso de peso as chances mais elevadas de insatisfação se apresentam naqueles com maior nível de escolaridade. Estudos realizados com mulheres no Brasil e no exterior também observaram maior prevalência de insatisfação por excesso de peso entre as de maior renda ${ }^{34,35}$. Uma possível explicação para esse resultado relaciona-se aos parâmetros do "corpo belo" nos distintos grupos sociais. Estudos qualitativos realizados na cidade do Rio de Janeiro mostram como mulheres residentes em bairros ricos e nas favelas apresentam visões diferentes do que é considerado um "corpo bonito" 36 .

Entre as mulheres casadas, a associação com a insatisfação por excesso de peso pode estar relacionada ao próprio envolvimento em uma relação afetiva, tendo em vista a interferência da relação com o outro na construção da sua própria imagem corporal, a expectativa do parceiro sobre o corpo da mulher, que pode ser explícita ou apenas um sentimento da própria mulher a respeito do parceiro e do seu próprio corpo $^{2,37}$. Outros estudos ${ }^{24,31}$ também encontraram associação entre a situação conjugal e a insatisfação com a imagem corporal por excesso de peso. Tom et al. ${ }^{38}$ observaram que a insatisfação com a imagem corporal é menos importante entre os casados e, segundo Meltzer et al..$^{39}$ cônjuges quando estão em um relacionamento estável e duradouro diminuem seus esforços na manutenção de seu peso por não verem como prioridade a atração do parceiro. Por outro lado, Lundborg et al. ${ }^{40}$ afirmam que os casados teriam maior preocupação com a forma do corpo, uma vez que isso pode afetar as relações do cônjuge. Esses estudos apontam que a relação afetiva é importante, mas não há consenso quanto ao seu efeito na insatisfação com a imagem corporal.

Associação entre atividade física e a insatisfação com a imagem corporal poderia ser explicada pelo desbalanceamento do gasto energético que contribui para o ganho de peso e aumenta a discrepância entre a imagem corporal atual e ideal ${ }^{41}$. O tipo de atividade física praticada pode estar associado à pretensão de um corpo ideal, sob a influência da mídia que apresenta corpos extremamente magros para as mulheres e com massa muscular bastante desenvolvida para os homens ${ }^{4,10}$. Outra possível explicação é que o maior cuidado e preocupação com o corpo po- 
Tabela 5. Fatores associados com a Imagem Corporal por excesso de peso segundo sexo. Estudo ELSA-Brasil, 2008-2010.

\begin{tabular}{|c|c|c|c|c|c|c|}
\hline \multicolumn{7}{|c|}{ Insatisfação com a Imagem Corporal por Excesso de Peso } \\
\hline \multirow[b]{2}{*}{ Variáveis } & \multicolumn{3}{|c|}{ Mulheres } & \multicolumn{3}{|c|}{ Homens } \\
\hline & $\begin{array}{c}\text { Bloco } 1 \\
\text { OR (IC95\%) }\end{array}$ & $\begin{array}{c}\text { Bloco } 2 \\
\text { OR (IC95\%) }\end{array}$ & $\begin{array}{c}\text { Modelo Final } \\
\text { OR (IC95\%) }\end{array}$ & $\begin{array}{c}\text { Bloco 1 } \\
\text { OR (IC95\%) }\end{array}$ & $\begin{array}{c}\text { Bloco } 2 \\
\text { OR (IC95\%) }\end{array}$ & $\begin{array}{c}\text { Modelo Final } \\
\text { OR (IC95\%) }\end{array}$ \\
\hline Idade & $1,02(1,01-1,03)^{\star *}$ & $1,02(1,0-1,03)$ & $1,02(1,0-1,03)$ & $1,01(1-1,02)$ & $1,0(0,99-1,01)$ & $1,0(0,99-1,02)$ \\
\hline \multicolumn{7}{|l|}{ Escolaridade } \\
\hline Ensino superior & 1 & - & 1 & 1 & - & 1 \\
\hline Ensino médio & $1,07(0,88-1,3)$ & - & $1,09(0,92-1,31)$ & $0,93(0,77-1,12)$ & - & $0,87(0,74-1,03)$ \\
\hline Ensino fundamental & $0,94(0,65-1,37)$ & - & $1,0(0,7-1,43)$ & $0,57(0,45-0,72)^{\star * *}$ & - & $0,53(0,43-0,65)^{* * *}$ \\
\hline \multicolumn{7}{|l|}{ Renda per capita } \\
\hline Alta & 1 & - & - & 1 & - & - \\
\hline Média & $1,0(0,82-1,23)$ & - & - & $1,05(0,87-1,28)$ & - & - \\
\hline Baixa & $1,16(0,91-1,48)$ & - & - & $0,98(0,78-1,22)$ & - & - \\
\hline \multicolumn{7}{|l|}{ Situação Conjugal } \\
\hline Solteiro & 1 & - & 1 & & - & - \\
\hline Separado/Viúvo & $1,28(1,0-1,64)$ & - & $1,28(1,0-1,64)$ & & - & - \\
\hline Casado & $1,51(1,21-1,89)^{* * *}$ & - & $1,49(1,19-1,87)^{* * *}$ & & - & - \\
\hline \multicolumn{7}{|l|}{ Atividade Física } \\
\hline Forte & & 1 & 1 & & 1 & 1 \\
\hline Moderada & & $0,9(0,64-1,25)$ & $0,87(0,62-1,23)$ & & $1,44(1,1-1,87)^{\star *}$ & $1,45(1,11-1,9)^{\star *}$ \\
\hline Fraca & & $1,56(1,18-2,08)^{\star *}$ & $1,52(1,14-2,03)^{* *}$ & & $1,86(1,51-2,3)^{* * *}$ & $1,99(1,62-2,45)^{* * *}$ \\
\hline \multicolumn{7}{|l|}{ Consumo Frutas } \\
\hline Alto & & 1 & 1 & & 1 & \\
\hline Diário & & $0,85(0,69-1,06)$ & $0,84(0,69-1,04)$ & & $1,12(0,89-1,4)$ & - \\
\hline Semanal & & $0,99(0,75-1,31)$ & $1,01(0,78-1,32)$ & & $1,16(0,91-1,48)$ & - \\
\hline Raro & & $0,72(0,52-0,98)^{\star}$ & $0,74(0,55-1,0)$ & & $1,01(0,78-1,32)$ & - \\
\hline \multicolumn{7}{|l|}{ Consumo Hortaliças } \\
\hline Alto & & 1 & - & & 1 & - \\
\hline Diário & & $0,94(0,74-1,2)$ & - & & $1,15(091-1,45)$ & - \\
\hline Semanal & & $1,04(0,77-1,39)$ & - & & $1,22(0,94-1,57)$ & - \\
\hline Raro & & $1,08(0,77-1,52)$ & - & & $1,1(0,84-1,45)$ & - \\
\hline \multicolumn{7}{|l|}{ Consumo Álcool } \\
\hline Não consome & & - & - & & 1 & 1 \\
\hline $\begin{array}{l}\text { Consumo } \\
\text { moderado }\end{array}$ & & - & - & & $1,19(1,02-1,39)^{\star}$ & $1,12(0,96-1,31)$ \\
\hline Consumo excessivo & & - & - & & $1,93(1,47-2,52)^{\star * *}$ & $1,89(1,44-2,47)^{* * *}$ \\
\hline \multicolumn{7}{|l|}{ Hábito de Fumar } \\
\hline Nunca fumou & & 1 & 1 & & 1 & 1 \\
\hline Ex-fumante & & $1,53(1,23-1,9)^{* * *}$ & $1,51(1,22-1,88)^{* * *}$ & & $1,2(1,01-1,42)^{\star}$ & $1,26(1,06-1,5)^{\star *}$ \\
\hline Fumante & & $0,86(0,68-1,1)$ & $0,86(0,67-1,09)$ & & $0,59(0,48-0,72)^{\star * *}$ & $0,64(0,52-0,79)^{\star * *}$ \\
\hline
\end{tabular}

Fonte: Elaborado pelas autoras.

deria aumentar o nível de atividade física e, por sua vez, um ciclo de busca incessante pela imagem de um corpo "perfeito" poderia aumentar a insatisfação com a imagem corporal. Entretanto, isso não parece ser consenso na literatura ${ }^{42}$.

A intensidade da atividade física pode impactar sua relação com imagem corporal entre os homens. Duas meta-análises apontaram que a atividade física vigorosa teve um impacto maior em comparação com a de intensidade leve ${ }^{43,44}$. No entanto, em meta-análise recente, Bassett-Gunter e colaboradores sugerem que a atividade física de intensidade baixa a moderada foi positivamente relacionada à imagem corporal entre os homens 
e não encontrou associação com a intensidade vigorosa. Por isso não há consenso de que a prática de atividades físicas fortes seja o caminho para atingir corpos volumosos ou alcançar o desejo de diminuir a quantidade de gordura corporal ${ }^{13}$. Esta relação pode ser uma explicação para a associação da prática de atividade física moderada e leve com chances mais elevadas de insatisfação com a imagem corporal por baixo peso e também por excesso de peso. Entretanto, deve-se levar em consideração as limitações do instrumento utilizado para quantificar a atividade física, que faz referência à intensidade e duração da atividade física semanal de lazer e de deslocamento, mas não quantifica há quanto tempo o indivíduo pratica aquele tipo de atividade física, informação que tem relação direta com as modificações corporais que a atividade física pode ocasionar ${ }^{16}$.

Santos Silva et al. ${ }^{24}$ não encontraram associação estatisticamente significativa entre as mulheres e a prática de atividade física; nos homens essa associação foi importante apenas entre os insatisfeitos com a imagem corporal por excesso de peso. Entretanto, a avaliação no estudo citado não diferenciou o tipo de atividade física praticada, classificando os sujeitos de forma dicotômica para a prática de atividades físicas no lazer. Nikniaz et al. ${ }^{34}$ concluíram que mulheres que superestimaram seu tamanho corporal tendem a participar em programas de atividade física com mais frequência. Isso pode ser devido ao fato de que a insatisfação com o corpo as tornam mais propícias a essa busca pelo desejo de atingir o seu ideal de corpo.

Frutas e hortaliças são componentes importantes de uma dieta saudável, por serem alimentos de baixa densidade energética em relação ao seu volume e favorecem a manutenção do peso corporal saudável ${ }^{45}$. Entretanto, neste estudo encontrou-se chances maiores de insatisfação com a imagem corporal por baixo peso e um consumo baixo de frutas (semanal), apenas entre as mulheres, o que pode estar relacionado a uma menor ingestão de calorias, que poderia acarretar baixo peso e a insatisfação observada. Outra possibilidade seria uma provável associação com transtorno de alimentação. No entanto, na população ELSA-Brasil a prevalência desse transtorno está em torno de $6 \%{ }^{46}$, enquanto outros estudos braileiros apontam uma prevalência entre 15 e $22 \%{ }^{47}$ em pacientes que procuravam tratamento para emagrecer. Além disso, Lucan $^{48}$ aponta que o benefício do consumo de frutas e hortaliças se dá através da substituição de alimentos não saudáveis. No entanto, esse efeito não se dá apenas com frutas e hortaliças, aconteceria também com o consumo de outros alimentos saudáveis como, por exemplo, alimentos integrais.

O consumo excessivo de álcool, entre os homens, esteve associado tanto à insatisfação com a imagem corporal por baixo peso quanto por excesso de peso. Este fato pode estar relacionado ao tipo de bebida alcoólica consumida. O consumo de bebidas com grande teor alcoólico, como cachaça, uísque e licores, pode estar associado ao baixo peso, por conta da substituição da alimentação pela bebida. Em contrapartida o consumo de bebidas com um teor alcoólico mais baixo, como a cerveja, pode estar associado a um maior aporte calórico por conta da própria bebida e também por alimentos que em geral são ingeridos como acompanhamento ${ }^{49,50}$. Realizouse uma análise de sensibilidade com a variável consumo de álcool por tipo de bebida. Os insatisfeitos por baixo peso apresentaram uma chance três vezes maior de consumo excessivo de bebidas alcoólicas destiladas e resultados não significativos para o consumo de vinho e cerveja/chope. Com relação à insatisfação por excesso de peso, a chance foi de 1,49 para o consumo excessivo de cerveja/chope (dados não apresentados).

$\mathrm{O}$ fato de ser ex-fumante se manteve associado a uma chance mais elevada de insatisfação com a imagem corporal por excesso de peso em ambos os sexos. Um estudo que avaliou a imagem corporal, sem fazer diferenciação na direção da insatisfação, observou uma associação entre o hábito de fumar e a insatisfação com a imagem corporal em adultos jovens universitários de ambos os sexos nos EUA ${ }^{51}$. Assim como neste estudo, aqueles que apresentaram o hábito de fumar estavam mais insatisfeitos com a sua imagem corporal, quando comparados aos não fumantes.

É importante ressaltar algumas limitações. Encontrou-se poucos estudos que tenham utilizado a escala de silhuetas de Kakeshita com 15 silhuetas, na população adulta, dentro da faixa etária explorada neste trabalho, provavelmente por conta da escala ter sido desenvolvida há 10 anos. Os estudos utilizados para comparação nesta discussão em geral utilizaram a escala de silhuetas de Stunkard. Esta possui um número menor de figuras, apenas nove silhuetas que não foram construídas com base no IMC, o que pode de certa forma limitar a comparação dos resultados. A forma de categorização dos resultados das escalas de silhuetas apresentada na literatura, naqueles sujeitos insatisfeitos com a imagem corporal são agregados no mesmo grupo independente do grau de insatisfação apresentado, ou 
seja, a diferença pode ser por apenas uma figura, ou por uma diferença de até 14 figuras no caso da escala usada neste estudo. Esta classificação pode, de certa forma, limitar os resultados, diminuindo a força da associação entre a insatisfação com a imagem corporal e as questões associadas. Destaca-se também que estes resultados decorrem de dados seccionais sendo possível apenas estabelecer associações entre o desfecho e as variáveis de exposição e não aferir uma relação de causalidade para explicar essas associações.

Apesar das limitações apresentadas, atualmente as escalas têm se mostrado como importantes instrumentos para avaliar a percepção da autoimagem e o grau de satisfação ${ }^{52}$. Além disso, esse método tem sido apontado como um instrumento de diagnóstico que permite identificar indivíduos e/ou populações em risco de desenvolverem transtornos alimentares ou atitudes e comportamentos que podem trazer resultados negativos para sua saúde ${ }^{53}$. Por se tratar de um estudo que envolve um grande número de variáveis, um ponto importante é o controle de qualidade realizado antes e durante a coleta de dados. Este é um ponto forte do ELSA-Brasil, que contou com vários procedimentos prévios (treinamento e padronização da equipe de examinadores e supervisores, préteste dos instrumentos e do manual de instruções, entre outros), e ao longo do estudo garantindo a confiabilidade e validade das informações analisa- das. Outro ponto forte foi o pequeno número de variáveis sem informação e as estratégias utilizadas para garantir a adesão ao estudo.

\section{Conclusão}

É importante ressaltar que encontraram-se diferenças importantes por sexo. Entre as mulheres, a menor escolaridade e o consumo de frutas semanalmente aumentou a chance de insatisfação por baixo peso, enquanto a prática de atividade física moderada reduziu. A insatisfação por estar acima do peso foi observada entre as casadas, as que praticam atividade física leve e as ex-fumantes. Entre os homens, os fatores importantes na insatisfação por baixo peso foram o nível médio de escolaridade, o consumo excessivo de álcool e a prática atividade física leve ou moderada. Por outro lado, a prática de atividade física leve e moderada também aumentaram a chance de insatisfação por excesso de peso. Esses achados contribuem para reforçar que hábitos e comportamentos não saudáveis podem influenciar a insatisfação com a imagem corporal. Assim, a atuação sobre os fatores possíveis de modificação, com campanhas que reforcem a importância de comportamentos saudáveis, pode contribuir para melhorar a percepção de um corpo saudável diminuindo a insatisfação com o próprio corpo.

\section{Colaboradores}

LS Albuquerque colaborou na concepção, análise formal, redação, revisão e edição. MJM Fonseca colaborou na concepção, análise formal, metodologia, redação, revisão e edição. RH Griep colaborou na metodologia, redação, revisão e edição. EML Aquino, LO Cardoso e D Chor colaboraram na revisão e edição. Todos os autores leram e concordaram com a versão publicada do manuscrito. 


\section{Referências}

1. Cash TF, Pruzinsky T. Body image: a handbook of science, practice, and prevention. $2^{\mathrm{a}}$ ed. New York, London: The Guilford Press; 2011.

2. Cash T. Cognitive-Behavioral Perspectives on Body Image. In: Cash T, editor. Encyclopedia of Body Image and Human Appearance. $1^{\mathrm{a}} \mathrm{ed}$. Amsterdam: Academic Press Elsevier; 2012. p. 334-342.

3. Rodgers RF, Salè P, Chabrol H. Psychological functioning, media pressure and body dissatisfaction among college women. Eur Rev Appl Psychol 2010; 60: 89-95.

4. Grogan S. Body image: understanding body dissatisfaction in men, women and children. $3^{\mathrm{a}}$ ed. Abingdon (United Kingdom): Routledge; 2017.

5. Barreto PS, Ferrandez AM, Guihard-Costa AM. Predictors of body satisfaction: differences between older men and women's perceptions of their body functioning and appearance. J Aging Health 2011; 23: 505528.

6. Carvalho PHB, Castro MR, Ferreira MC. Imagem Corporal e vida Adulta. In: Ferreira MEC, Castro MR, Morgado FFDR, editores. Imagem Corporal: reflexões e diretrizes e práticas de pesquisa. $1^{\text {a }}$ ed. Juiz de Fora: Editora UFJF; 2014. p. 87-113.

7. Grogan, S. Body Image Development in Adulthood. In: Cash TF, Smolak L, editors. Body Image: a handbook of science, pratice, and prevention. 2 ed. New York: The Guilford Press; 2011.p. 93-100.

8. McLean SA, Paxton SJ. Body Image in the Context of Eating Disorders. Psychiatr Clin North Am 2019 Mar;42(1):145-156.

9. Pereira EF, Teixeira CS, Gattiboni BD, Bevilacqua LA, Confortin SC, Silva TR. Percepção da imagem corporal e nível socioeconômico em adolescentes: revisão sistemática. Rev Paul Pediatr 2011; 29(3):423-429

10. Karazsia BT, Crowther JH. Social body comparison and internalization: mediators of social influences on men's muscularity-oriented body dissatisfaction. Body Image 2009;6(2):105-112.

11. Guðnadóttir $\mathrm{U}$, Garðarsdóttir RB. The influence of materialism and ideal body internalization on bodydissatisfaction and body-shaping behaviors of young men and women: support for the Consumer Culture Impact Model. Scand J Psychol 2014; 55(2):151-159.

12. Kim Y, Austin SB, Subramanian SV, Kawachi I. Bodyweight perception, disordered weight control behaviors, and depressive symptoms among Korean adults: The Korea National Health and Nutrition Examination Survey 2014. PLoS One 2018; 13(6):e0198841.

13. Bassett-Gunter R, McEwan D, Kamarhie A. Physical activity and body image among men and boys: A meta-analysis. Body Image 2017; 22:114-128.

14. Costa LCF, Vasconcelos FAG. Influência de fatores socioeconômicos, comportamentais e nutricionais na insatisfação com a imagem corporal de universitárias Rev Bras Epidemiol 2010; 13(4): 665-576.

15. Pelegrini A, Sacomori C, Santos MC, Sperandio FF, Cardoso FL. Body image perception in women: prevalence and association with anthropometric indicators. Rev Bras Cineantropom Desempenho Hum 2014; 16(1):58-65.
16. Coelho CG, Giatti L, Molina MD, Nunes MA, Barreto SM.Body Image and Nutritional Status Are Associated with PhysicalActivity in Men and Women: The ELSA-Brasil Study. Int J Environ Res Public Health 2015; 12(6):6179-6196.

17. Silva PO, Guimarães JMN, Griep RH, Melo ECP, Matos SMA, Molina MDC, Barreto SM, Fonseca MJMF. Association between Body Image Dissatisfaction and Self-Rated Health, as Mediated by Physical Activity and Eating Habits: Structural Equation Modelling in ELSA-Brasil. Int. J Environ Res Public Health 2018; 15(4):790.

18. Leite HM, Garcez A, Nunes MAA, Pattussi MP, Canuto R, Paniz VMV, Olinto, MTA. Shift work, sleep duration, and body image dissatisfaction among female workers in southern Brazil. Arch Womens Ment Health 2018; 1:1-10.

19. Poltronieria TS, Tussetb C, Gregolettoc MLO, Cremonese C. Insatisfação com a imagem corporal e fatores associados em mulheres do sul do Brasil. Cienc Saude 2016; 9(3):128-134.

20. Aquino EM, Barreto SM, Bensenor IM, Carvalho MS, Chor D, Duncan BB, Lotufo PA, Mill JG, Molina Mdel C, Mota EL, Passos VM, Schmidt MI, Szklo M. Brazilian Longitudinal Study of Adult Health (ELSA -Brasil): objectives and design. Am J Epidemiol 2012; 175(4):315-24.

21. Kakeshita IS, Silva AIP, Zanatta DP, Almeida SS. Construção e fidedignidade teste-reteste de escalas de silhuetas brasileiras para adultos e crianças. Psicologia: Teoria e Pesquisa 2009; 25(2):263-70

22. Griep RH, Aquino EM, Chor D, Kakeshita IS, Gomes AL, Nunes MA. Confiabilidade teste-reteste de escalas de silhuetas de autoimagem corporal no Estudo Longitudinal de Saúde do Adulto. Cad Saude Publica 2012; 28(9):1790-1794.

23. Tribess S, Virtuoso Junior JS, Petroski EL. Nutritional status and perceived body image of elderly women in the Northeast of Brazil. Cien Saude Colet 2010; 15(1):31-38

24. Santos Silva DA, Nahas MV, Sousa TF, Del Duca GF, Peres KG. Prevalence and associated factors with body image dissatisfaction among adults in southern Brazil: a population-based study. Body Image 2011; 8(4):427-431.

25. Anschutz DJ, Engels RC, van Strien T. Susceptibility for thin ideal media and eating styles. Body Image 2008; 5(1):70-79.

26. Anschutz DJ, Engels RC, Becker ES, van Strien T. The bold and the beautiful. Influence of body size of televised media models on body dissatisfaction and actual food intake. Appetite 2008; 51(3):530-537.

27. Chor D, Cardoso LO, Nobre AA, Griep RH, Fonseca MJM, Giatti L, Bensenor I, Molina MDCB, Aquino EML, Diez-Roux A, Castiglione DP, Santos SM. Association between perceived neighbourhood characteristics, physical activity and diet quality: results of the Brazilian Longitudinal Study of Adult Health (ELSA-Brasil). BMC Public Health 2016; 8:(16):751. 
28. Piccinelli M, Tessari E, Bortolomasi M, Piasere O Semenzin M, Garzotto N, Tansella M. Efficacy of the alcohol use disorders identification test as a screening tool for hazardous alcohol intake and related disorders in primary care: a validity study. BMJ 1997; 314:420-424.

29. Word Health Organization (WHO). Obesity: preventing and managing the global epidemic. Report of a WHO Consulation on Obesity. Geneva: WHO; 1998.

30. Alvarenga MS, Philippi ST, Lourenço BH, Sato PM, Scagliusi FB. Insatisfação com a imagem corporal em universitárias brasileiras. J Bras Psiquiatr 2010; 59(1):44-51.

31. Silva VS, Souza I, Silva DAS, Fonseca MJM. Prevalence and factors associated with overweight in adults - Brazil, 2008-2009. Rev. Bras. Cineantropom. Desempenho Hum 2014; 16(2):161-170

32. Fernandes MGM, Garcia LG. O corpo envelhecido: percepção e vivência de mulheres idosas. Interface 2010; 14(35):879-890.

33. Fin TC, Portella MR, Scortegagna SA. Velhice e beleza corporal das idosas: conversa entre mulheres. Rev Bras Geriatr Gerontol 2017; 20(1):77-87.

34. Nikniaz Z, Mahdavi R, Amiri S, Ostadrahimi A, Nikniaz $\mathrm{L}$. Factors associated with body image dissatisfaction and distortion among Iranian women. Eat Behav 2016; 22:5-9.

35. Kops NL, Bessel M, Knauth DR, Caleffi M, Wendland EM. Body image (dis)satisfaction among low-income adult women. Clin Nutr 2018; 38(3):1-5.

36. Novaes FV. Com que corpo eu vou? sociabilidade e usos do corpo nas mulheres das camadas altas e populares. Rio de Janeiro: Ed. PUC-Rio e Pallas; 2009.

37. Jones DC. Interpersonal and Familial Influences on the Development of Body Image. In: Cash TF, Smolak L, editors. Body image: a handbook of science, practice, and prevention. $2^{\mathrm{a}} \mathrm{ed}$. New York: The Guilford Press; 2011.p. 110-118.

38. Tom G, Chen A, Liao H, Shao J. Body image, relationships, and time. J Psychol 2005; 139(5):458-468.

39. Meltzer AL, Novak SA, McNulty JK, Butler EA, Karney BR. Marital satisfaction predicts weight gain in early marriage. Health Psychol 2013; 32(7): 824-827.

40. Lundborg P, Nystedt P, Lindgren B. Getting ready for the marriage market? The association between divorce risks and investments in attractive body mass among married Europeans. J Biosoc Sci 2007; 39(4):531-544.

41. Liao Y, Harada K, Shibata A, Ishii K, Oka K, Nakamura Y, Sugiyama T, Inoue S, Shimomitsu T. Joint associations of physical activity and screen time with overweight among Japanese adults. Int J Behav Nutr Phys Act 2011; 30(8):131.

42. Homan K. Athletic-ideal and thin-ideal internalization as prospective predictors of body dissatisfaction, dieting, and compulsive exercise. Body Image 2010; 7(3):240-245.

43. Hausenblas H, Fallon EA. Exercise and body image: A meta-analysis. Psychol Health 2006; 21(1):33-47.
44. Reel JJ, Greenleaf C, Baker WK, Aragon S, Bishop D Cachaper C, Handwerk P, Locicero J, Rathburn L, Reid WK, Hattie J. Relations of body concerns and exercise behavior: a meta-analysis. Psychol Rep 2007;101(3 Pt 1):927-942.

45. Jaime PC, Figueiredo ICR, Moura ECd, Malta DC. Fatores associados ao consumo de frutas e hortaliças no Brasil, 2006. Rev Saude Publica 2009; 43(Supl. 2):5764.

46. Gralle APBP, Moreno AB, Juvanhol LL, Fonseca MJF, Melo ECP, Nunes MAA, Toivanen, S, Griep RH. Job strain and binge eating among Brazilian workers participating in the ELSA-Brasil study: does BMI matter? J Occup Health 2017; 59: 247-255.

47. Azevedo AP, Santos CC, Fonseca DC. Transtorno da compulsão alimentar periódica. Rev Psiquiatr Clin 2004; 31(4):170-172.

48. Lucan SC. Fruit-and-vegetable consumption may not be inadequate. Am J Public Health 2012; 102(10):e3; author reply e3-4.

49. Cibeira GH, Muller C, Lazzaretti R, Nader GA, Caleffi M. Consumo de bebida alcoólica, fatores socioeconômicos e excesso de peso: um estudo transversal no sul do Brasil. Cienc Saude Colet 2013; 18(12):3577-3584.

50. Lukasiewicz E, Mennen LI, Bertrais S, Arnault N, Preziosi P, Galan P, Hercberg S. Alcohol intake in relation to body mass index and waist-to-hip ratio: the importance of type of alcoholic beverage. Public Health Nutr 2005; 8(3):315-320.

51. Clark MM, Croghan IT, Reading S, Schroeder DR, Stoner SM, Patten CA, Vickers KS.The relationship of body image dissatisfaction to cigarette smoking in college students. Body Image 2005; 2(3):263-270.

52. Moraes C, Anjos LA, Marinho SM. Development, adaptation and validation of silhouette scales for self-assessment of nutritional status: a systematic review. Cad Saude Publica 2012; 28(1):7-20.

53. Côrtes MG, Meireles AL, Friche AA, Caiaffa WT, Xavier CC. Silhouette scales and body satisfaction in adolescents: a systematic literature review. Cad Saude Publica 2013; 29(3):427-44.

Artigo apresentado em 11/10/2018

Aprovado em 24/07/2019

Versão final apresentada em 26/07/2019

Editores-chefes: Romeu Gomes, Antônio Augusto Moura da Silva 
